$8^{\text {th }}$ U. S. National Combustion Meeting

Organized by the Western States Section of the Combustion Institute

and hosted by the University of Utah

May 19-22, 2013

\title{
Self Induced Buoyant Blow Off in Upward Flame Spread on Thin Solid Fuels
}

\author{
Michael C. Johnston ${ }^{1}$, James S. T'ien ${ }^{1}$, Derek E.Muff ${ }^{1}$,Sandra L. Olson ${ }^{2}$, Paul V. Ferkul ${ }^{3}$ \\ ${ }^{1}$ Case Western Reserve University, Cleveland, $\mathrm{OH}$ \\ ${ }^{2}$ NASA Glenn Research Center, Cleveland, $\mathrm{OH}$ \\ ${ }^{3}$ National Center for Space Exploration Research, Cleveland, $\mathrm{OH}$
}

\begin{abstract}
Upward flame spread experiments were conducted on a thin fabric cloth consisting of $75 \%$ cotton and $25 \%$ fiberglass. The sample is sandwiched symmetrically with stainless steel plates with the exposed width varying between 2 to $8.8 \mathrm{~cm}$ from test to test and $>1.5 \mathrm{~m}$ tall. The bottom edge was ignited resulting in a symmetric two sided flame. For the narrower samples $(\leq 5 \mathrm{~cm})$, two sided flame growth would proceed until reaching some limiting value $(15-30 \mathrm{~cm}$ depending on sample width). Fluctuation or instability of the flame base on one side would initially become visible and then the flame base would retreat downstream and cause extinguishment on one side. Detailed examination of the still images shows that the fuel continues to vaporize from the extinguished side due to the thermally thin nature of the fuel. But, due to the remaining inert fiberglass mesh, which acts as a flashback arrestor, the extinguished side was not able to be reignited by the remaining flame. The remaining flame would then shrink in length due to the reduced heat transfer to the solid to a shorter length. The one-sided flame will spread stably with a constant speed and a constant flame length to the end of the sample. A constant length flame implies that the pyrolysis front and the burnt out fronts move at the same speed. For the wider samples $(\geq 7 \mathrm{~cm})$, no one-sided extinction is observed. Two-sided flames spread all the way to the top of the sample. For these wider widths, the flames are still growing and have not reached their limiting length if it exists.
\end{abstract}

\begin{abstract}
Care was taken to minimize the amount of non-symmetries in the experimental configuration. Repeated tests show that blow-off can occur on either side of the sample. The flame growth is observed to be very symmetric during the growth phase and grew to significant length $(>10 \mathrm{~cm})$ before extinction of the flame on one side. Our proposed explanation of this un-usual phenomenon (i.e. stronger two-sided flame cannot exist but weaker one-sided flame can) is as follows: The observed one-sided extinction is a blow-off induced by buoyant entrainment. It is known that the flammable diffusion flame regime is bounded by quenching and blow-off limits when varying incoming air velocity. The narrowest samples tested (between 2 and $5 \mathrm{~cm}$ ) begin within the flammable range, but as the flame grows, the buoyancy driven air velocity increases at the neighborhood of the flame base. The initially stable flame crosses the extinguishment boundary resulting in a flame blow-off. When one-side of the flame extinguishes, the remaining side shrinks due to the reduced heat transfer to the solid. This reduces the induced velocity and the flame becomes stable. It is proposed that this may have implications to upward flame growth beyond this experiment.
\end{abstract}

\section{Introduction}

The characteristics of upward flame spread are of importance to material flammability and fire safety research. The upward spread configuration has been used as a metric for qualifying materials for safety purposes such as in NASA STD-6001 Test \#1 [1]. For flat samples, Test \#1 utilizes a fixed geometry with exposed sample width set at $5 \mathrm{~cm}$ and height set at $30 \mathrm{~cm}$. The sample 
is ignited at the base using a fixed energy chemical ignition source or hotwire. If the material ignites and spreads further than 6 inches $(15.24 \mathrm{~cm})$, it fails to qualify for use aboard the space station or NASA spacecrafts.

In terrestrial applications, upward spread represents a hazardous burning mode because of its faster growth/spread rate. A standard test in this configuration is UL94 [2]. Note that NASA STD 6001test\#1 is similar to UL94V in many respects.

It has been shown previously that sample width can have a significant effect on the upward flame spread characteristics including flame size, heat generation rate, and spread rate [3-6]. Width should also affect the flame extinction limits. In this work, we conducted upward flame spread tests in normal gravity using a special type of composite fabric. Several sample widths were used. An unexpected but very interesting phenomenon, i.e. self induced flame extinction, was observed in many of the tests. The observation and a proposed interpretation are given below.

\section{Experimental set up}

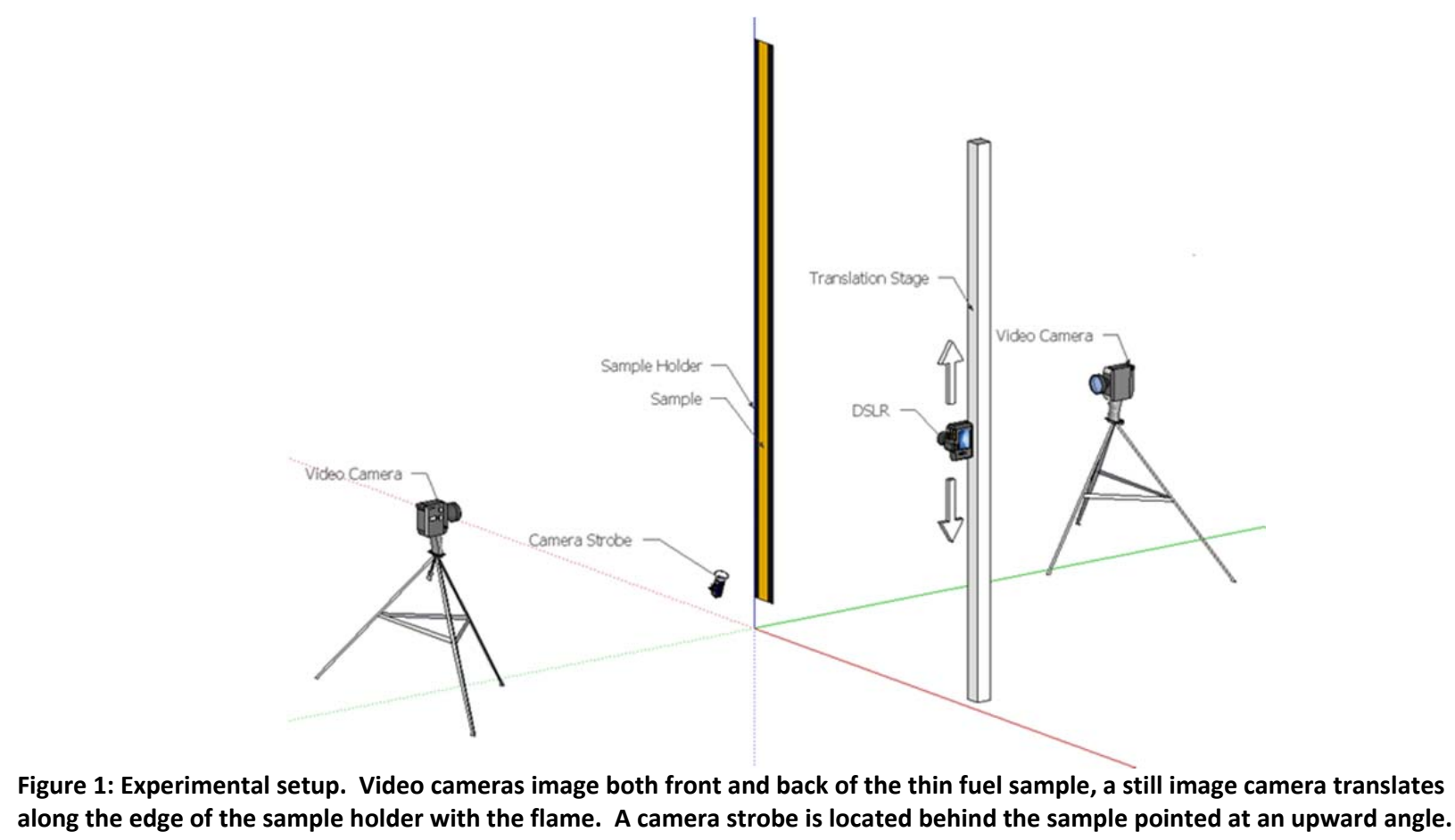




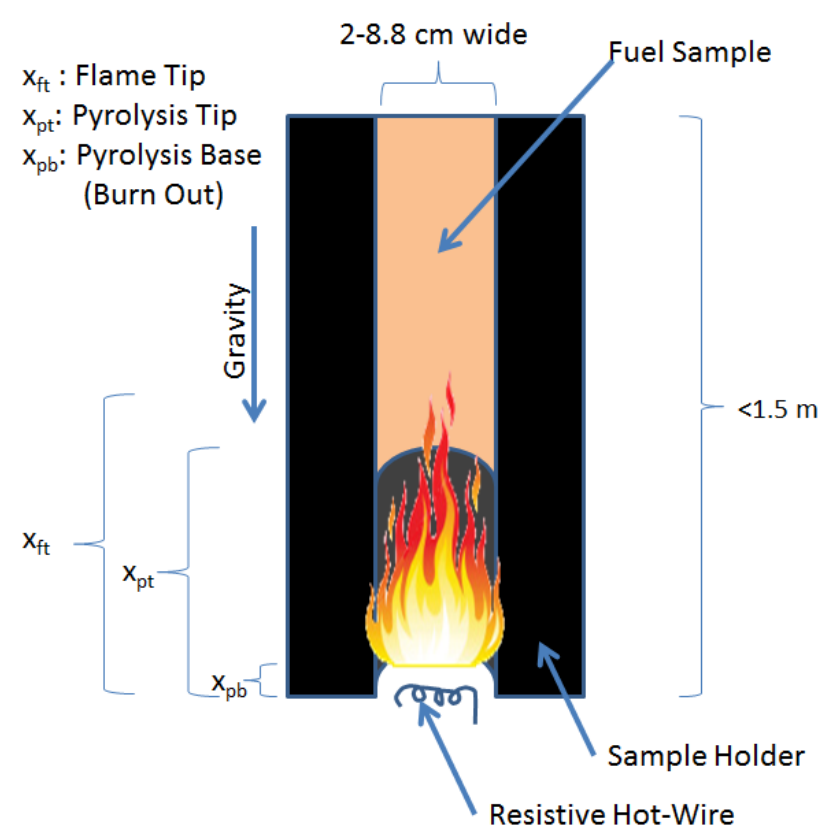

Figure 2: The definitions of flame tip, pyrolysis tip, and pyrolysis base are given in the diagram.

The experiment configuration mimics that of the NASA STD-6001 Test 1 . The thin fuel is sandwiched between parallel stainless steel sample holders .035 ” thick x 2.5 ” wide with adjustable width $2-8.8 \mathrm{~cm}$ between the plates to accommodate various fuel widths and height up to $1.5 \mathrm{~m}$. The fuel used in this experiment is unique. It is made from a simple weave fabric consisting of thread spun with $75 \%$ cotton and $25 \%$ fiberglass strands. As the cotton burns away, the fiberglass component of the thread is left behind maintaining the fuels structural integrity. This inert matrix simplifies the burning characteristics of the fuel by preventing tearing, ripping, and curling of the solids surface as would happen with a burning material such as paper. A comparison of this fuel with other materials is given in [7].

The custom made fabric fuel (referred to as SIBAL named after the original experiment it was originally designed for [8]) has been studied in a large number of careful laboratory scale experiments in a variety of environmental conditions [7-9] and the fuel itself is more fully described in [Ref]. The area density of the fabric is $.0181 \mathrm{~g} / \mathrm{cm}^{2}$ (remembering that $75 \%$ by weight is 
combustible cellulose, and $25 \%$ is inert fiberglass). The pre-burned SIBAL fuel can be seen in Figure 3a and after the flame front has passed, the remaining inert matrix is shown in Figure 3b.

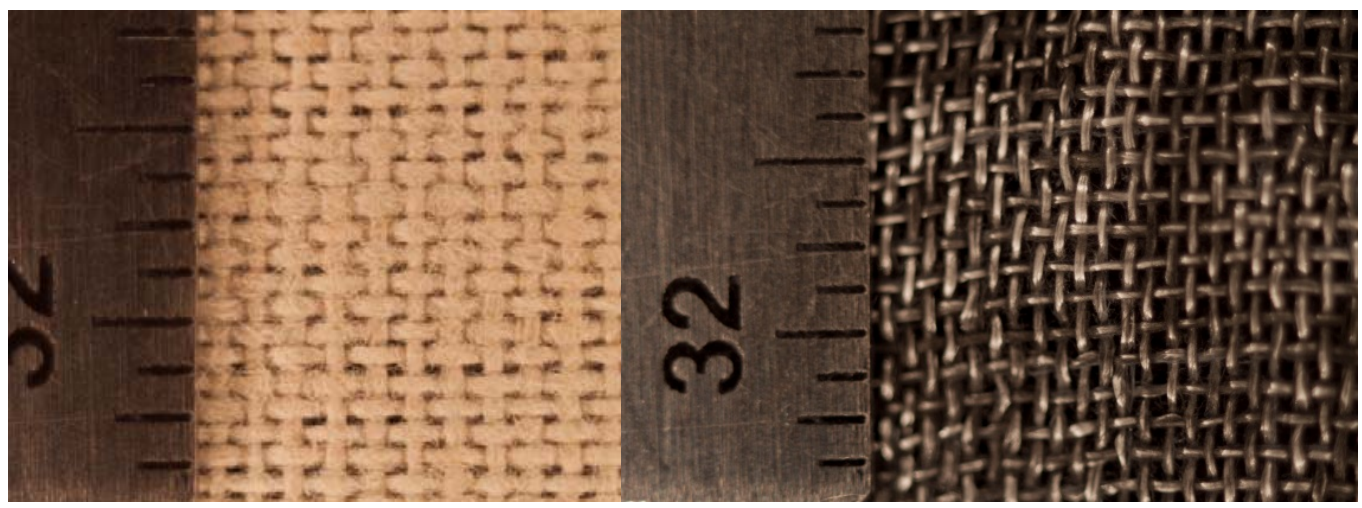

Figure 3: a.) Unburned SIBAL fabric, b.) Inert fiberglass matrix after the flame has passed

The leftover fiberglass matrix has also been found to act as a flame barrier since the gaps between the threads are smaller than the quenching distance of the flame. This allows for the somewhat unique possibility of a one sided flame existing on a thin fabric fuel [9]. Note also that this fuel sample is sufficiently thin so that, in most experiments, it behaves as a thermally thin specimen.

Fuel ignition was achieved using a Kanthal hotwire in a sawtooth pattern alternating on the front and back of the fuel surface at the bottom edge of the fuel sample.

The material is imaged with 1080p high definition video cameras perpendicular to the front and back surfaces of the fuel which image at 30 frames per second. A third high resolution digital SLR still camera zoomed in to the size of the flame views the fuel and sample holder from the edge and moves along a track parallel to the flame propagation. The still camera is capable of shooting an 8 frame burst in approximately 1 second. A strobe light located on the opposite side of the sample holder illuminates the smoke present and captures the instantaneous smoke field. The still camera needs to integrate over approximately $1 / 30-1 / 60^{\text {th }}$ of a second to capture the flame. However, the strobe which captures the smoke field is on the order of $1 / 1000^{\text {th }}$ of a second. 


\section{Experimental Results}

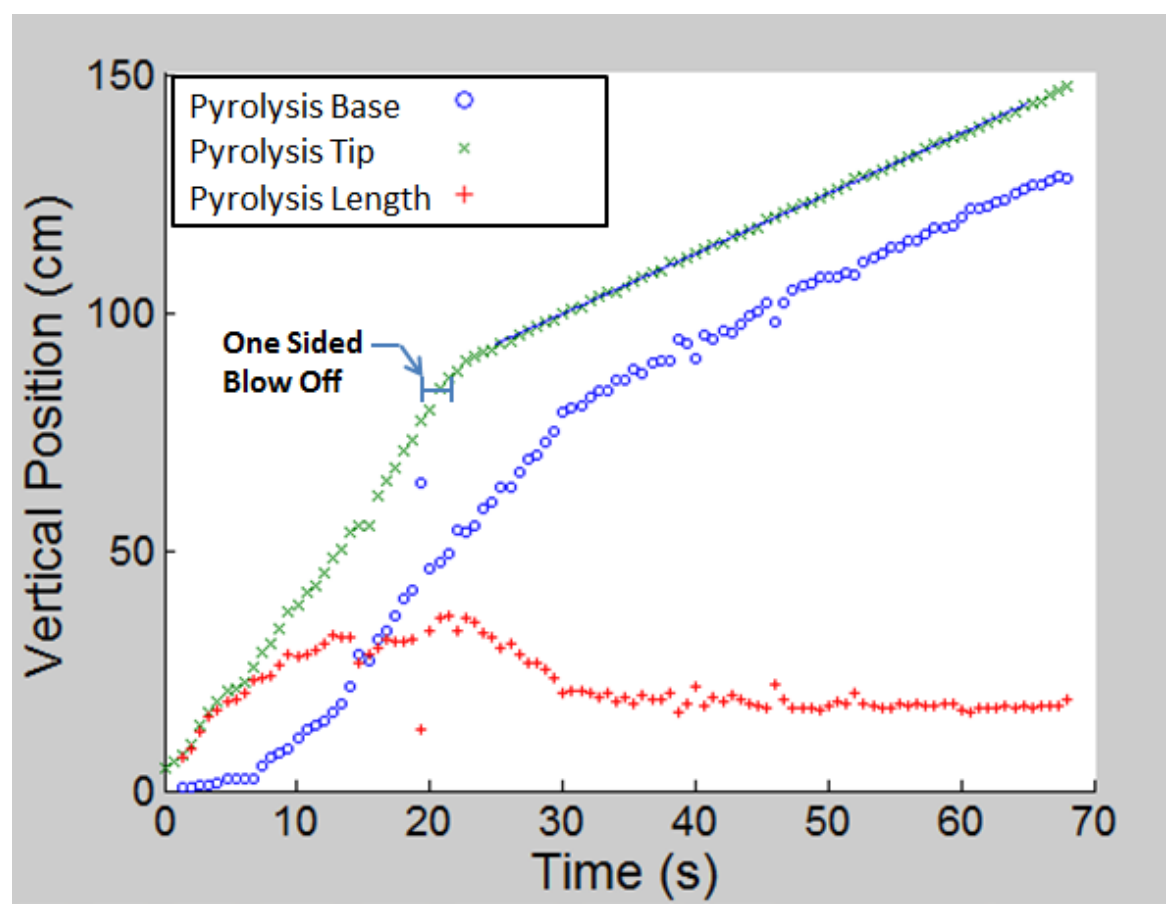

Figure 4: $5 \mathrm{~cm}$ wide case with one sided blow-off extinction

Figure 4 shows a typical case of flame ignition, growth, and upward spread on our thin fabric fuel for a 5-cm wide sample. The position of pyrolysis front and burn out front are plotted with respect to time on the abscissa. In the very early stages, the pyrolysis tip can be seen to propagate downstream at an increasing rate (curve is concave upward). Due to the physically thin nature of the fabric used, the amount of solid fuel available to pyrolyze is limited and flame base moves upward when the combustible is consumed. Note that during the initial growth stage, the flame tip will accelerate upwards while the flame base lags behind, remaining at the axis origin until the fuel has a chance to burn out. At some time depending on conditions, the burn out front (and therefore the flame base) will propagate upward. If the fuel burnout rate catches up the pyrolysis front rate, a constant limiting length flame is reached and a steady spread with a constant spread rate results. In many normal gravity upward tests with wide samples, steady spread may not be observed for the entire length of the sample. Steady spread with a limiting flame length were easier to find narrow 
samples, in low pressure environments, in partial gravity and in concurrent low-velocity flame spread in microgravity [10-13].

In the present work, it was observed from the video, one side of the flame is extinguished (blown-off) during the flame growth as indicated in Figure 4 for the 5-cm wide sample. This happens at t 20s when the pyrolysis length is about $35 \mathrm{~cm}$ long. The flame on the other side, however, stays but quickly shrinks in length. Responding to this flame blow-off and lengthshrinking event, the rate of pyrolysis front propagation decreases following by a decrease of the fuel burnout rate as shown in Fig. 4 (with some time delay). The one-sided flame then reaches a limiting length and spreads steadily all the way to the end of the sample.
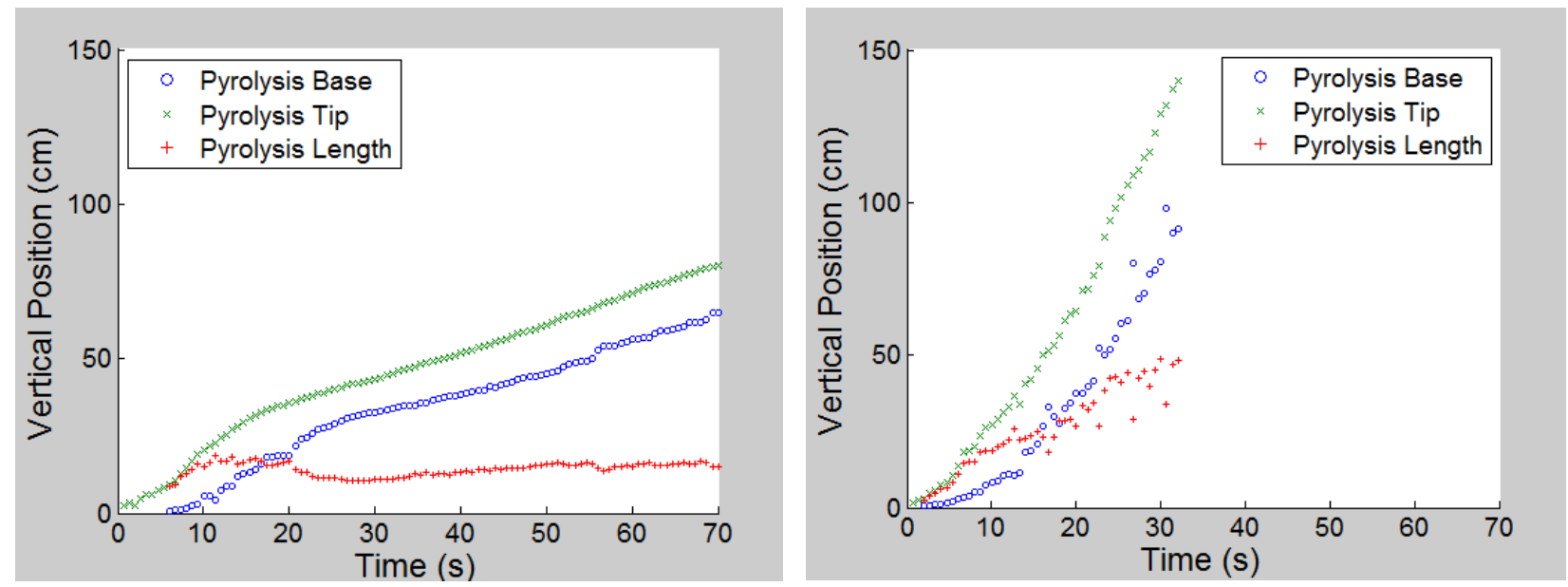

Figure 5 a.) $2 \mathrm{~cm}$ wide Sibal, b.) $7.5 \mathrm{~cm}$ wide Sibal Fuel

The 2-cm wide sample test shown in Figure 5a exhibits a qualitatively similar trend as in Fig. 4 for the 5-cm sample. The critical pyrolysis length for the one-sided blow off to occur, however, is shorter $(\sim 15 \mathrm{~cm})$. Both the initial flame growth rate and the final one-side steady spread rates are lower than the 5-cm case. Consistently, the steady pyrolysis length is also shorter.

For the 7.5-cm wide sample, Fig. 5(b) shows both the prolysis front growth rate and the pyrolysis length increase with time continuously over the entire sample. There is no one-sided 
extinction. No steady spread is observed. The entire spread duration is in the growth phase. Tests with $8.8 \mathrm{~cm}$ sample width show similar a trend.

These upward tests have be repeated many times. Since the one-side extinction was observed for the narrower samples (2-5 cm widths), much care was taken to eliminate non-symmetries in the experimental configuration and in the environment. Nevertheless, repeated tests show that extinguishment can occur on either side of the sample. After ignition, the initial flame growth is observed to be very symmetric on both sides of the sample. One-sided extinction occurs only when the flame reaches a critical length (e.g. $\sim 15 \mathrm{~cm}$ for 2 -cm sample and $\sim 35 \mathrm{~cm}$ for 5 -cm sample). The flame extinction therefore is not an ignition anomaly nor non-symmetry of the experimental setup. We believe that the observed extinction is a true physical phenomenon in buoyant flames. The next section is devoted to the explanation.

\section{Proposed mechanism of self-induced flame extinction.}

The central question is why the flame extinguishes itself when its length becomes too large? Why the shorter and weaker flame on the other side of the sample remains?

Before going into our interpretation, let us examine a detailed sequence of photo images of the flame growths-extinction-spread events. Fig. 6 shows the edge-view pictures of the $5 \mathrm{~cm}$ case from Figure 4. The camera is translating with the flame in consecutive images to keep the flame in view. The time stamps are shown at the top of each photo.

In Fig. 6a, a well established two sided flame is already present. The strobe light can be seen in the bottom of the frame, but as the camera translates, it will leave the field of view. In frame b, the flame base on the right hand side of the fuel sample is beginning to retreat downstream. Frame c shows the right hand side flame base continuing to retreat but unburned pyrolyzate continuing to 
leave the fuel surface. Frame d shows the right hand side flame nearing extinction, the flame is propagating in to a region where the fuel surface is not adequately preheated, the retreating flame has blown off.

The extinguished side of the fuel sample will not reignite due to the inert mesh left behind which acts as a flame barrier. Frame e shows the large amount of fuel vapor which continues to enter the gas phase, but remains unburnt. The absence of flane heat feedback from the extinguish side shortens the flame on the remaining side. One may regard this as equivalent to an increase of sample thickness (since the flame is only on one side). The shortened flame shows good stability and is able to spread upward all the way to the end of the sample. So again, why does the longer (stronger) flame extinguish but the shorter (weaker) flame stay?

To answer this question, we first examine the extinction mechanism of a diffusion flame, specifically for a spreading solid diffusion flame in concurrent flow. Fig. 7 (a) shows several flammability boundaries using ambient oxygen percentage as the ordinate and flow velocity as the abscissa. Different flamability boundaries represents different sample widths. Each boundary consists of two branches: a high velocity blow-off branch and a low-velocity quenching branch [12]. In a fixed ambient oxygen environment, quenching occurs when the oxygen supply rate becomes too low and the weak low-intensity flame loses a large percentage of energy due to radiation and conduction. This is an active area of research interest in microgravity combustion. On the other end, high-velocity extinction is a flame stabilization problem. When the air velocity near the flame stabilization zone becomes too large, the flow residence time in the reaction initiation zone becomes too small ( or in nondimensional term the Damkohler based on stabilization zone size is too small), and the flame can not be stabilized. The reaction zone is blown off downstream. In this extinction mode, the near-limit flame is a high intensity strong flame since the air velocity and oxygen supply 
rate is high. Note that this critical air velocity is at the flame stabilization zone where fuel vapor first meets the upstream oxygen. In the upward spreading flame configuration, it is at the bottom flame base as indicated in Fig. 6. In a purely forced system, the velocity magnitude at the stabilization zone is controlled by the upstream condition. In upward flame, the velocity at the base is induced by gravity. The buoyant velocity magnitude at the base can be affected by the size of the flame. Within certain limits and sensitivity, it is expected that a longer flame will induce a larger velocity at the base.

The U-shaped flammability boundary computed in Ref. 13 is for two-dimensional flames (very wide samples). It is expected that sample width will affect the flammability limits with smaller flammability domain for narrower samples. This is illustrated in the flammability map in Fig. 7(a) for three sample widths. Note that Fig 7 is used to explain extinction mechanisms. It is not a quantitive plot.

In normal earth gravity, the buoyant-induced flow velocity at the flame base is large enough so extinction is located on the flame blowoff side. Using flame stabilization zone length as a scale, the induced velocity $\sim 25 \mathrm{~cm} / \mathrm{s}$. Since this is the smallest length in the flame, this is the minimum buoyant induced velocity at the flame base. The computed flow velocity at the lowest oxygen point (the dividing point between blow-off and quenching branches) is around $10 \mathrm{~cm} / \mathrm{s}$ [13]. So flame extinction in this upward burning configuration in normal gravity is by blowoff. For blowoff , it is also expected that the critical blowoff velocity is larger for wider samples as illustrated in Fig. 7a. This coupled with a previous statement that a longer flame will induce a larger flow velocity at the flame base is sufficient to explain the obseved self-induced extinction phenomena in upward spread, to be detailed next. 
For 21\% oxygen, the flame blowoff velocity boundaries for three sample widths are shown Fig. 7(b) using a finer scale. With time as the ordinate, the burning history of three samples are qulitatively traced. For the $5 \mathrm{~cm}$ sample, trace $A$ starts with a small induced velocity at the flame base. As the flame grows in length, the bouyant velocity increases and eventually exceeds the critical blowoff velocity, the flame on one side is blown off (marked by $\mathrm{x}$ in the graph). The absence of the flame on one side leads to a shorter flame on the remaining side (roughly $1 / 2$ shorter). The buoyant induced velocity on this side at the base is reduced below the blowoff limit and the flame is stabilized. The shorter one-sided flame reaches a limiting length for steady upward spread.

Trace $B$ for the $2 \mathrm{~cm}$ case in Fig.7(b) is similar to trace $A$ except the critical blowoff velocity is smaller and the flame is shorter. For the $7.5 \mathrm{~cm}$ sample, trace $C$ shows that the blowoff velocity limit is larger and the buoyant induced velocity at the base does not cross over the limit. The flame continues to grow until reaching the end of the sample.

\section{Concluding remarks}

A very interesting flame extinction mode has been found in upward spread over a solid fuel. The upward spreading flame blows off when its length becomes too big. An explanation is offerred based on increased buoyant induced velocity at the flame base stabilization zone. Although this is observed using a special type of thin solid, it could be a more general near-limit phenomenon.

Although there is no direct mesurement of the buoyant-induced velocities in our experiment due to the experimental complexity necessary for flow field measurements, we believe computer computer models can help support our proposed mechanism in future work. 

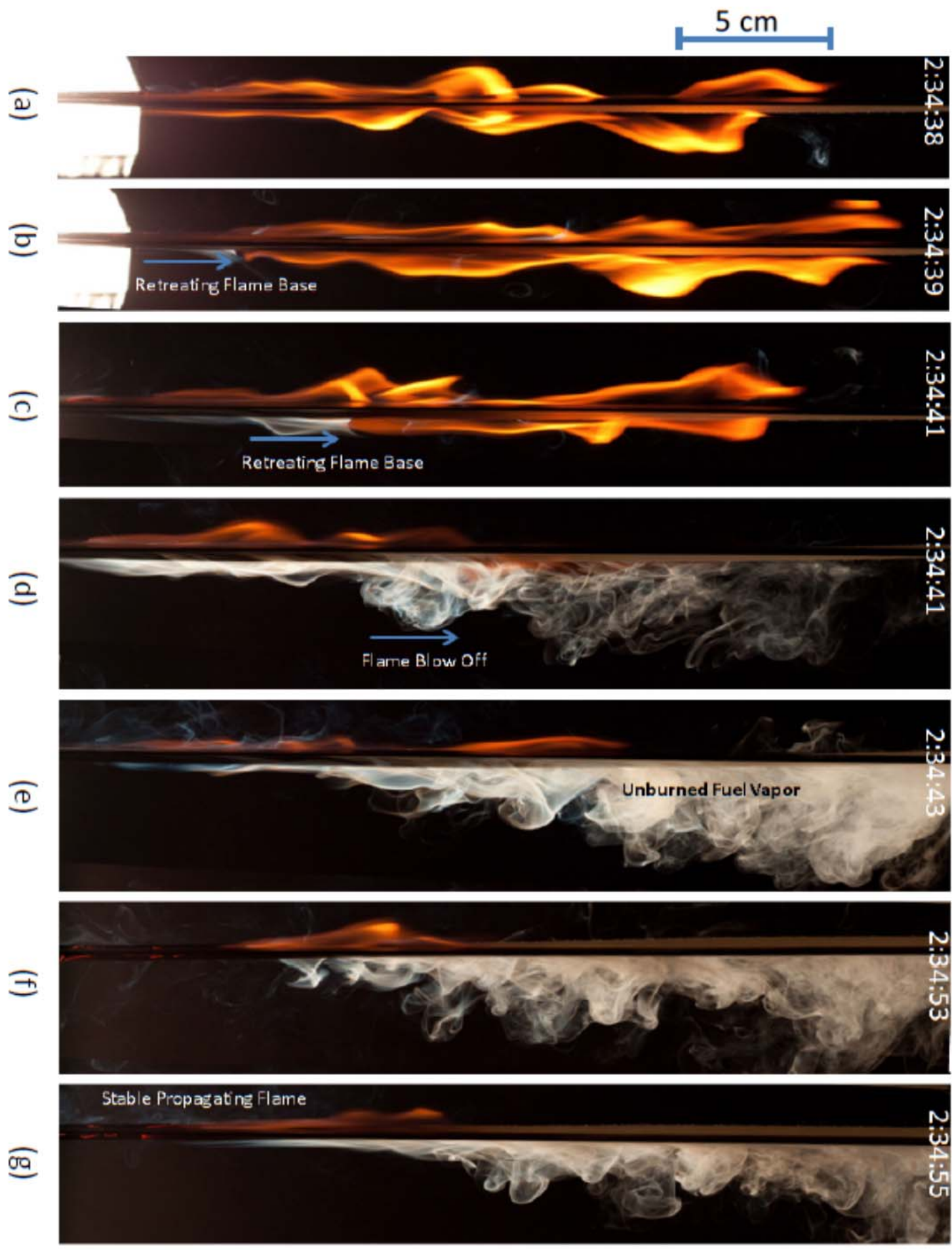

Figure 6: One sided extinguishment from the edge view is shown. Gravity is toward the left. a.) A two sided flame is established b.) the right hand side (RHS) flame begins retreating downstream, c.) RHS flame is continuing to retreat while pyrolysis is maintained, d.) RHS flame blows off, e.) Unburned fuel vapor on the right hand side continues to escape, f.) The left hand side flame shrinks due to the reduced heat flux to the solid, $g$.) the left hand side flame reaches a steady length and will propagate to the end of the sample. 

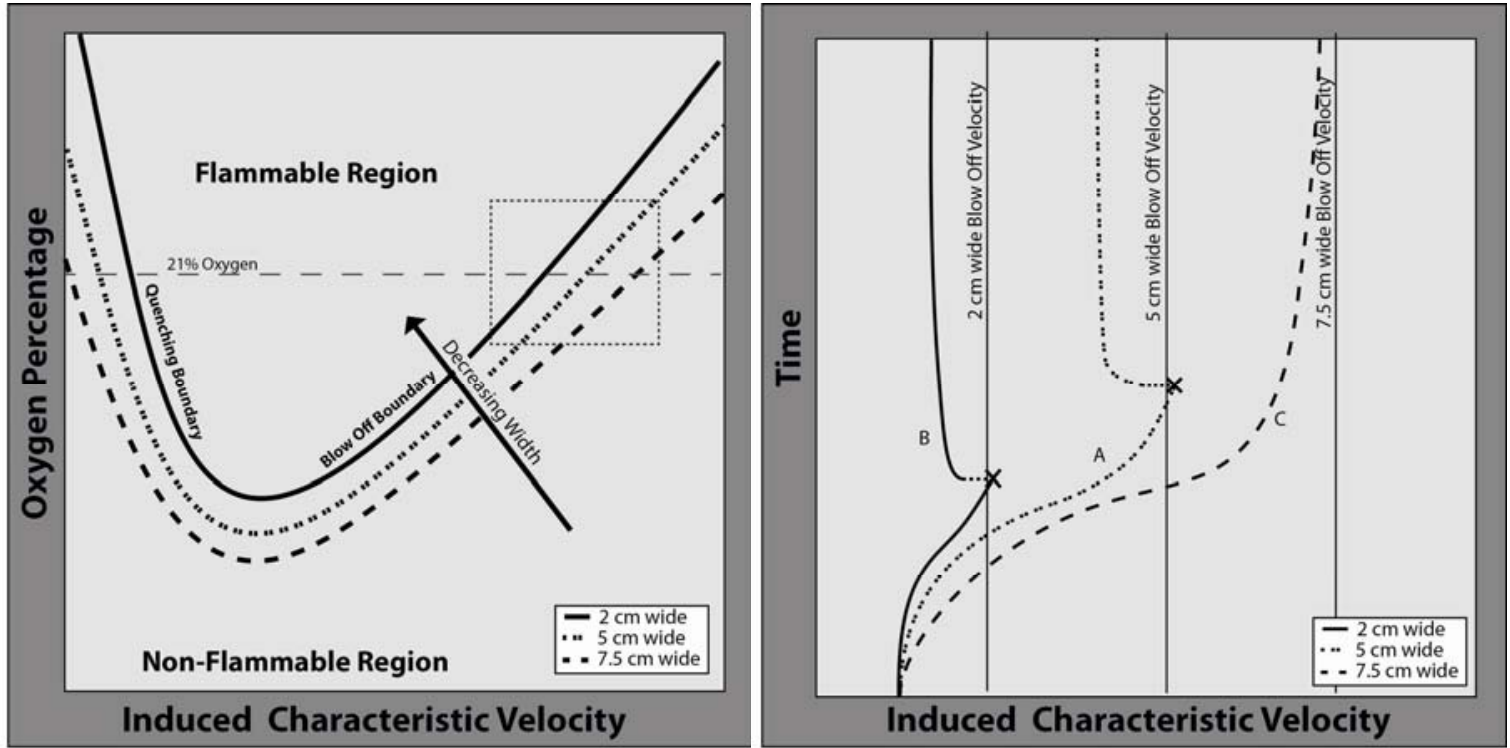

Figure 7: A) Qualitative flammability map bounded by quenching and blow off limits predicted for various fuel widths. B): Qualitative curve of the characteristic entrained air velocity in the flame stabilization zone plotted with respect to time. 


\section{Acknowledgements}

This research was funded by a grant from NASA. JST and DEM also would like to acknowledge the support from Underwriters Laboratories.

\section{References}

1. "Flammability, Odor, Offgassing, and Compatibility Requirements and Test Procedures for Materials in Environments that Support Combustion," NASA, NASA-STD-6001, 1998.

2. UL-94. "Standard for Tests for Flammability of Plastic Materials for Parts in Devices and Appliances”. October, 1996.

3. Tsai Kuang-Chung, "Width Effect on Upward Flame Spread," Fire Safety Journal, vol. 44, pp. 962-967, June 2009.

4. Linton K. Honda and Paul D. Ronney, "Mechanisms of Concurrent-Flow Flame Spread Over Solid Fuel Beds," Proceedings of the Combustion Institute, vol. 28, pp. 2793-2801, 2000.

5. W. E. Mell and T. Kashiwagi, "Effects of Finite Sample Width On Transition and Flame Spread in Microgravity," Proceedings of the Combustion Institute, vol. 28, pp. 2785-2792, 2000.

6. Ali S. Rangwala, Steven G. Buckley, Jose L. Torero, "Upward flame spread on a vertically oriented fuel surface: The effect of finite width”, Proceedings of the Combustion Institute, vol. 31, pp. 2607-2615, 2007.

7. Julie Kleinhenz, "The Flame Spread and Extinction Characteristics of Cotton-Fiberglass Fabric," Case Western Reserve University, Cleveland, Master Thesis, 2002.

8. Ferkul, P.V., Kleinhenz, J., Shih, H.-Y., Pettegrew, R., Sacksteder, K. and T’ien, J.: Solid Combustion Experiments Using a Continuous Fuel Dispenser for Use in Microgravity and Associated Model Computations, Microgravity Science and Technology: XV/2: 3-12, 2004.

9. J Kleinhenz, P Ferkul, R Pettegrew, K Sacksteder, and J T'ien, "One-sided Flame Spread Phenomena of Thermally Thin Composite Cotton/Fiberglass Fabric," Fire and Material, vol. 29, pp. 23-37, 2005.

10. Chu, L., Chen, C. H., and T’ien, J. S.: “Upward Propagation Over Paper Samples,” ASME Paper , 81WA/HT-42, 1981.

11. Feier, I.I., Shih, H.-Y., Sacksteder, K.V. and T’ien, J.S.: Upward Flame Spread Over Thin Solids in Partial Gravity, Proceedings of the Combustion Institute 29: 2569-2577, 2002.

12. Ferkul, P.V., Olson, S.L., Johnston, M.C., Tien, J.S.: "Flammability Aspects of Fabric in Opposed and Concurrent Air Flow in Microgravity”, $8^{\text {th }}$ US National Meeting, Combustion Institute, University of Utah, May 2013.

13. Ferkul, P. V. and T’ien, J. S.: “A Model of Low-Speed Concurrent Flow Flame Spread Over a Thin Fuel,” Combustion Science and Technology, Vol. 99, No. 4-6, p. 345-370 (1994). 